\title{
SCHAUDER DECOMPOSITIONS IN $(m)$
}

\author{
DAVID W. DEAN
}

1. Introduction. If the Banach space $B$ is a countably infinite direct sum of nontrivial subspaces $B_{i}, B=\sum B_{i}$, such that each $b$ has a unique representation $b=\sum b_{i}, b_{i} \in B_{i}$; and if each of the projections $P_{i} b=b_{i}$ is continuous then say that $B$ has a Schauder decomposition (a brief history of this topic is found in [8]). Sanders [9] has shown that $(m)$, the space of bounded sequences, does not have a Schauder decomposition such that the coordinate sequences $\left(\delta_{i j}\right)_{j=1}^{\infty}$ lie in distinct $B_{k}$. The purpose of this note is to prove $(m)$ has no Schauder decomposition whatsoever. In fact this is true for a class of spaces which include those $C(H)$ spaces for which $H$ is compact, Hausdorff, and extremely disconnected; (and all direct factors of such spaces (the $P_{\lambda}$-spaces) (see [1, pp. 94-96] for a discussion of such $C(H)$ ).

Let $B^{*}$ be the conjugate space of $B$ and let $\left(f_{n}\right)$ be a sequence in $B^{*}$. If $\lim _{n} f_{n}(b)$ exists and is finite for every $b$ in $B$ then the limit defines an element of $B^{*}[2$, p. 52]. The following properties of $(m)$ are critical for our proof.

(1) If $\lim f_{n}(b)=f(b)$ exists for each $b$ in $(m)$ then $\lim F\left(f_{n}\right)$ exists for each $F$ in $(m)^{* *}$ and is equal to $F(f),[3$, pp. 168, 169]. That is, sequential weak* convergence in $(m)^{*}$ implies weak convergence.

(2) Let $X, Y$ be Banach spaces and let $S, T$ be weakly compact linear operators such that

$$
X \stackrel{S}{\rightarrow}(m) \stackrel{T}{\rightarrow} Y .
$$

Then $T S: X \rightarrow Y$ is compact [2, p. 494].

Replacing $(m)$ with $B$ in (1) and (2) our main theorem is: If $B$ has properties (1) and (2) then it does not have a Schauder decomposition.

2. The main theorem. Suppose now that $B$ has property (1) and a Schauder decomposition $\sum B_{j}$. Then $b=\sum_{1}^{\infty} P_{j}(b)$ for each $b$ in $B$. Define conjugate projections $Q_{j}$ in $B^{*}$ by $Q_{j} f(b)=f\left(P_{j} b\right)$ for every $b$ in $B, f$ in $B^{*}$. Each sum $\sum_{n}^{m} P_{j}$ is a continuous projection and has conjugate projection $\sum_{n}^{m} Q_{j}$. The set of projections $\sum_{n}^{m} P_{j}$ is uniformly norm bounded (in $m, n$ ) since $\left(\sum_{n}^{m} P_{j}\right)(b)=\sum_{n}^{m} b_{j} \rightarrow 0$, $n, m \rightarrow \infty\left[2\right.$, p. 52]. As $\left\|\sum_{n}^{m} Q_{j}\right\|=\left\|\sum_{n}^{m} P_{j}\right\|$ the set of projections

Presented to the Society, April 4, 1966; received by the editors February 21, 1966. 
$\sum_{n}^{m} Q_{j}$ is uniformly norm bounded as well. Moreover for each $f$ in $B^{*}$ one has

$$
\left(\sum_{n}^{m} Q_{j} f\right)(b)=\sum_{n}^{m} f\left(P_{j} b\right)=f\left(\sum_{n}^{m} P_{j} b\right) \rightarrow 0, \quad n, m \rightarrow \infty .
$$

Then, using (1), $\sum_{1}^{\infty} Q_{j} f$ converges weakly to $f$. It is easy to see that the sequence $\left(Q_{j} f\right)$ is unique. Thus, in this sense, $B^{*}$ has a weak decomposition $B^{*}=\sum_{1}^{\infty} B_{j}^{\prime}$, where $B_{j}^{\prime}=Q_{j} B^{*}$. In fact $\sum B_{i}^{\prime}$ is a Schauder decomposition of $B^{*}$ ((i, ii) below).

Proposition. If $B$ has a Schauder decomposition and satisfies (1) then

(i) If $b_{j} \in B_{j}$ for each $j$ and either $b_{j}=0$ or $\left\|b_{j}\right\|=1$ then $\left\{b_{j} \mid b_{j} \neq 0\right\}$ is a basis for $\left[b_{j}\right]$ (the closed span of $\left(b_{j}\right)$ ).

(ii) If $f_{j} \in B_{j}^{\prime}$ for each $j$ and either $f_{j}=0$ or $\left\|f_{j}\right\|=1$ then $\left\{f_{j} \mid f_{j} \neq 0\right\}$ is a basis for $\left[f_{j}\right]$.

(iii) $\sum B_{j}^{\prime}$ is a Schauder decomposition of $B^{*}$.

(iv) $\left[f_{j}\right]$ in (ii) is reflexive.

(v) Let $F \in B^{* *}\left(=\left(B^{*}\right)^{*}\right)$ and let $F_{n}$ be $F$ restricted to $\sum_{n}^{\infty} B_{j}^{\prime}$. Then $\left\|F_{n}\right\| \rightarrow 0$ (the decomposition is shrinking in the sense of [8]).

(vi) $\left[b_{j}\right]$ in (i) above is reflexive.

Parts (iv) and (vi) are those needed to prove the main theorem, the others being steps to achieving (iv) and (vi).

Suppose now the proposition is known and suppose $B$ satisfies (1), (2), and has Schauder decomposition $\sum B_{j}$. Choose $b_{j} \in B_{j}$ with $\left\|b_{j}\right\|=1$. Let $B^{*}=\sum B_{j}^{\prime}$ be the decomposition in (iii). Choose $c$ such that $\left\|Q_{j}\right\| \leqq c$ for every $j$. Select $g_{j}$ having norm one such that $\left|g_{j}\left(b_{j}\right)\right|$ $\geqq 1 / 2$. Then $\left|Q_{j} g_{j}\left(b_{j}\right)\right| \geqq 1 / 2$ while $\left\|Q_{j} g_{j}\right\| \leqq c$. Set $f_{j}=Q_{j} g_{j} /\left\|Q_{j} g_{j}\right\|$. Then $1=\| f_{j}|\geqq| f_{j}\left(b_{j}\right) \mid \geqq 1 / 2 c>0$. Find such $f_{j}$ in $B_{j}^{\prime}$ for each $j$. Recall that if $i \neq j$ then $f_{j}\left(b_{i}\right)=0\left(Q_{j} f_{j}\left(P_{i} b_{i}\right)=f_{j}\left(P_{j} P_{i} b_{i}\right)=f_{j}(0)\right)$. Consider the mapping

$$
\left[b_{j}\right] \stackrel{I}{\rightarrow} B \stackrel{T}{\rightarrow}\left[f_{j}\right]^{*}
$$

where $I$ is the identity and $T$ is defined by $\operatorname{Tb}\left(\sum t_{j} f_{j}\right)=\sum t_{j} f_{j}(b)$. Then $I$ and $T$ are continuous and linear. Since by (iv) and (vi) both $\left[b_{j}\right]$ and $\left[f_{j}\right]$ are reflexive $\left[f_{j}\right]^{*}$ is also, and so $I$ and $T$ are weakly compact, $\left[1\right.$, p. 56]. Thus by (2) $T I$ is compact. Now $T I b_{j}\left(f_{j}\right)=T b_{j}\left(f_{j}\right)$ $=f_{j}\left(b_{j}\right)$ so $\left\|T I b_{j}\right\| \geqq 1 / 2 c$. A subsequence $T I b_{n_{j}}$ converges to an element of $\left[f_{j}\right]^{*}$ while $T I b_{n_{j}}$ converges weakly to 0 since

$$
T I b_{n_{j}}\left(\sum_{1}^{\infty} t_{i} f_{i}\right)=\sum_{1}^{\infty} t_{i} f_{i}\left(b_{n_{j}}\right)=t_{n_{j}} f_{n_{j}}\left(b_{n_{j}}\right) \stackrel{j}{\rightarrow} 0
$$


and in a reflexive space weak and weak* convergence are the same $\left[1\right.$, p. 25]. Thus $T I b_{n_{j}}$ converges in norm to 0 which is a contradiction to $\left\|T I b_{j}\right\| \geqq 1 / 2 c$ for all $j$.

3. Proof of the proposition. We shall need two well known theorems about bases, the first due to James [4], and the second to Karlin [5] (see $\left[1\right.$, p. 69] for a proof). A basis $\left(b_{n}\right)$ for $B$ is boundedly complete if $\left\|\sum_{1}^{n} t_{j} b_{j}\right\| \leqq K$ for all $n$ implies $\sum_{1}^{\infty} t_{j} b_{j}$ converges. It is shrinking if, for each $f$ in $B^{*} f_{n}=f \mid\left[b_{j}, j \geqq n\right]$ ( $f$ restricted to the closed span of $\left.\left\{b_{j} \mid j \geqq n\right\}\right)$ converges to $0\left(\left\|f_{n}\right\| \rightarrow 0\right)$.

(J) Let $B$ have basis $\left(b_{n}\right)$. Then $B$ is reflexive if and only if $\left(b_{n}\right)$ is shrinking and boundedly complete.

A sequence $\left(b_{n}\right)$ is a weak Schauder basis if for each $b$ in $B$ there is a unique sequence $\left(t_{j}\right)$ such that $\sum t_{j} b_{j}$ converges weakly to $b$ and if the functionals $\phi_{i}(b)=t_{i}$ are continuous.

(K) A weak Schauder basis is a basis.

Both (i) and (ii) will follow from (iii) and its proof. To show (iii), that is that $\sum B_{j}^{\prime}$ is a Schauder decomposition, we must show $f=\sum Q_{j} f$, for every $f$ in $B^{*}$. Now $\sum_{1}^{n} Q_{j} f$ converges weakly* to $f$ and so weakly to $f$. Thus each $f$ has a unique representation $\left(Q_{j} f\right)$ such that $\sum_{1}^{n} Q_{j} f$ converges weakly to $f$ and the coordinate projection $Q_{j}$ is continuous for each $j$. Such a decomposition we may call a weak decomposition. The following lemma was observed first by Ruckle [7, Theorem 1.20 and a remark on p. 549].

Lemma. If $X=\sum_{1}^{\infty} X_{j}$ is a weak decomposition then it is a Schauder decomposition.

Proof. Let $\sum_{1}^{\infty} x_{j}$ converge weakly to $x, x_{j} \in X_{j}$ for each $j$. Let $y_{j}=x_{j} /\left\|x_{j}\right\|$ if $x_{j} \neq 0$ and $y_{j}=0$ if $x_{j}=0$. In $\left[y_{j}\right]$ let $z_{n}=\sum_{j=1}^{\infty} t_{j n} y_{j}$ converge to $u$ as $n \rightarrow \infty$. Then, since each projection $R_{j} x=x_{j}$ is continuous $n: t_{j n} y_{j} \rightarrow u_{j}$ so that $t_{j n}$ converges, say to $t_{j}$, if $y_{j} \neq 0$ (and if $y_{j}=0$ we may set each $\left.t_{j n}=0\right)$. Moreover $u_{j}=t_{j} y_{j}$. The sequence $\left(t_{j}\right)$ is unique and $\sum_{1}^{n} t_{j} y_{j}=\sum_{1}^{n} u_{j}$ converges weakly to $u$. Thus $\left(y_{j}\right)$ is a weak basis for $\left[y_{j}\right]$ and by $(\mathrm{K})$ it is a basis (this proves (i) then).

Now the representation of $x$ in $\left[y_{j}\right]$ is $\sum_{1}^{\infty}\left\|x_{j}\right\| y_{j}=\sum_{1}^{\infty} x_{j}$ which converges to $x$ as $\left(y_{j}\right)$ is a basis. Thus the decomposition is a Schauder decomposition (hence (ii) follows from (i) in our particular case). We remark that the argument proves such a sequence $\left(y_{j}\right)$ is a basis for $\left[y_{j}\right]$ for any space $X$ having a Schauder decomposition $\sum X_{j}$.

(iv) To show $\left[f_{j}\right]$ in (ii) is reflexive we show its unit ball is weakly compact $[1$, pp. 51,56$]$. Let $\sum_{i=1}^{\infty} t_{j n} f_{j}=g_{n} \in\left[f_{j}\right]$ and $\left\|g_{n}\right\| \leqq 1$ for each $n$. Use a diagonal process to find a subsequence $g_{n_{i}}=h_{i}=\sum s_{j i} f_{j}$ such 
that $s_{j i} \rightarrow s_{j}$ as $i \rightarrow \infty$ for each $j$ (the projections $\left(Q_{j}\right)$ are uniformly norm bounded so this may be done).

By (iii) $\sum_{1}^{\infty} Q_{j}$ converges to the identity in the strong operator topology $\left[1\right.$, p. 34] so that the $\operatorname{set}\left(\sum_{m}^{\infty} Q_{j}\right)$ is uniformly norm bounded, say by $c$. Then for each $x$ in $B$

$$
\begin{aligned}
\left|h_{n}(x)-h_{m}(x)\right| \leqq & \sum_{1}^{k}\left(s_{j n} f_{j}-s_{j m} f_{j}\right)\left(x_{j}\right)|+| \sum_{k+1}^{\infty}\left(s_{j n} f_{j}-s_{j m} f_{j}\right)\left(x_{j}\right) \mid \\
\leqq & \left|\sum_{1}^{k}\left(s_{j n} f_{j}-s_{j m} f_{j}\right)\left(x_{j}\right)\right| \\
& +2 c\left|\sum_{k+1}^{\infty} x_{j}\right|<\left|\sum_{1}^{k}\left(s_{j n} f_{j}-s_{j m} f_{j}\right)\left(x_{j}\right)\right|+\epsilon
\end{aligned}
$$

if $K$ is large, $<2 \epsilon$ if $n, m$ are large. Thus $h_{n}$ is weakly* Cauchy and so weakly Cauchy. Since $h_{n}\left(x_{i}\right) \rightarrow s_{i} f_{i}\left(x_{i}\right)$ as $n \rightarrow \infty$ for every $x_{i}$ in $B_{i}$ one has that $h_{n}(x) \rightarrow \sum s_{i} f_{i}(x)$ for every $x$ in $B$. But then $h_{n}$ converges weakly to $\sum s_{i} f_{i}$ in $B^{*}$. Since $\sum s_{i} f_{i}$ is in $\left[f_{j}\right]$ the unit ball of $\left[f_{j}\right]$ is weakly compact.

(v) Let $F$ be in $B^{* *}$ and $F_{n}=F \mid \sum_{n} B_{j}^{\prime}$. Suppose $\left\|F_{n}\right\| \rightarrow 0$. Then $\exists \epsilon>0$ and a subsequence $\left(F_{n_{i}}\right)$ of $\left(F_{n}\right)$ such that $\left\|F_{n_{i}}\right\|>\epsilon$ for each $i$. If $n_{i}>m$ then $F_{m}=F_{n_{i}}$ on the domain of $F_{n_{i}}$ so that $\left\|F_{m}\right\| \geqq\left\|F_{n_{i}}\right\|$. Hence $\left\|F_{n}\right\|>\epsilon$ for every $n$. Choose $f_{1}=\sum_{1}^{n_{1}} g_{j}$ such that $1 \geqq\left\|f_{1}\right\| \geqq 1 / 2$ and $F_{1}\left(f_{1}\right)>\epsilon$. Choose $f_{2}=\sum_{n_{1}+1}^{n_{2}} g_{j}$ such that $1 \geqq\left\|f_{2}\right\| \geqq 1 / 2$ and $F_{n_{1}+1}\left(f_{2}\right)>\epsilon$. Continuing in this way we choose a sequence $F_{n_{j+1}}$ and $f_{j}=\sum_{n_{j-1}+1}^{n_{j}} g_{j}$ such that $1 \geqq\left\|f_{j}\right\| \geqq 1 / 2$ and $F_{n_{j-1}+1}\left(f_{j}\right)>\epsilon \quad\left(n_{0}=0\right)$. Now $\left[g_{j}\right]$ is reflexive so by $(\mathrm{J}) F \mid\left[g_{i} \mid i \geqq n_{j}\right]$ converges to 0 in norm. But $F\left(f_{j}\right)=F_{n_{j-1}+1}\left(f_{j}\right)>\epsilon$ for each $j$ contradicts $f_{j} \in\left[g_{j} \mid j \geqq n_{j-1}+1\right]$ for large $j$.

(vi) To show $\left[b_{j}\right]$ in (i) is reflexive we prove that $\left[b_{j}\right]^{*}$ has a basis which is both shrinking and boundedly complete and apply $(\mathrm{J})$. Choose $f_{j}$ in $B_{j}$ such that $f_{j}\left(b_{j}\right)=1$. The identity $I:\left[b_{j}\right] \rightarrow B$ has conjugate $I^{*}$ such that $I^{*} f_{j}=\phi_{j}$ is a biorthogonal sequence to $\left(b_{j}\right)$. Given any $\phi$ in $\left[b_{j}\right]^{*}$ there is a unique sequence $\left(t_{j}\right)$ such that $\phi(x)=\sum t_{j} \phi_{j}(x)$ for each $x$ in $\left[b_{j}\right]$ (let $\phi\left(b_{j}\right)=t_{j}, x=\sum s_{j} b_{j}$, then $\phi(x)=\sum t_{j} s_{j}$ $\left.=\sum t_{j} \phi_{j}(x)\right)$. Let $I^{*} g=\phi$ and $g=\sum g_{j}$. Then $I^{*} g_{j}=t_{j} \phi_{j}$ for some $t_{j}$ since $I^{*} g_{j}\left(\sum s_{i} b_{i}\right)=s_{j} g_{j}\left(b_{j}\right)=g_{j}\left(b_{j}\right) \phi_{j}\left(\sum s_{i} b_{i}\right)$. Thus $t_{j}=g_{j}\left(b_{j}\right)$. Thus $I^{*}\left(\sum g_{j}\right)=\sum t_{j} \phi_{j}$ converges. By the calculation preceding, it must converge to $\phi$ so that $\left(\phi_{j}\right)$ is a basis for $\left[b_{j}\right]^{*}$. If $\left\|\sum_{1}^{n} t_{j} \phi_{j}\right\| \leqq K$ for each $n$ then

$$
\sum_{n}^{m} t_{j} \phi_{j}\left(\sum s_{i} b_{i}\right) \stackrel{n m}{\longrightarrow} 0
$$


or $\sum_{1}^{n} t_{j} \phi_{j}$ converges weakly* to a limit $\phi$ and $\phi\left(b_{j}\right)=t_{j}$. Thus $\phi=\sum t, \phi_{j}$ so that

$$
\sum_{1}^{n} t_{j} \phi_{j} \stackrel{n}{\rightarrow} \sum t_{j} \phi_{j}
$$

Thus $\left(\phi_{j}\right)$ is boundedly complete and it remains to show it is shrinking. Let $F \in\left[\phi_{j}\right]^{*}$ and let $F_{n}\left(\sum t_{j} \phi_{j}\right)=F\left(\sum_{n}^{\infty} t_{j} \phi_{j}\right)$ for every $\sum t_{j} \phi_{j}$ in $\left[\phi_{j}\right]$. Then $\left\|F_{n}\right\| \rightarrow 0$ if and only if $\left\|F \mid\left[\phi_{j} \mid j \geqq n\right]\right\| \rightarrow 0$ as follows. The projections $\sum_{1}^{\infty} t_{j} \phi_{j} \rightarrow \sum_{n}^{\infty} t_{j} \phi_{j}$ are uniformly norm bounded, say by $K$. Then $\left\|F\left|\left[\phi_{j} \mid j \geqq n\right]\|\leqq\| F_{n}\|\leqq K\| F\right|\left[\phi_{j} \mid j \geqq n\right]\right\|$. Now $I^{* *} F_{n}=I^{* *} F$ on $\sum_{n}^{\infty} B_{j}^{\prime}$ and 0 on $\sum_{1}^{n-1} B_{j}^{\prime}$. Then, as above, $\left\|I^{* *} F_{n}\right\| \rightarrow 0$ if $\left\|I^{* *} F \mid \sum_{n}^{\infty} B_{j}^{\prime}\right\| \rightarrow 0$ which is (v). Since $\left\|I^{* *} F_{n}\right\|=\left\|F_{n}\right\|\left(I^{* *}\right.$ is an isometry) the basis is shrinking.

\section{BIBLIOGRAPHY}

1. M. M. Day, Normed linear spaces, Springer, Berlin, 1958.

2. N. Dunford and J. T. Schwartz, Linear operators, Vol. 1, Interscience, New York, 1958.

3. A. Grothendieck, Sur les applications lineaires faiblement compactes d'espaces $d u$ type $C(K)$, Canad. J. Math. 5 (1953), 129-173.

4. R. C. James, Bases and reflexivity of Banach spaces, Ann. of Math. (2) 52 (1950), 518-527.

5. S. Karlin, Bases in Banach spaces, Duke Math J. 15 (1948), 971-985.

6. C. W. McArthur and J. R. Retherford, Uniform and equicontinuous Schauder bases of subspaces, Canad. J. Math. 17 (1965), 207-212.

7. W. H. Ruckle, The infinite sum of closed subspaces of an F-space, Duke Math. J. 31 (1964), 543-554.

8. B. L. Sanders, Decompositions and reflexivity in Banach spaces, Proc. Amer. Math. Soc. 16 (1965), 204-208.

9. - On the existence of Schauder decompositions in Banach spaces, Proc. Amer. Math. Soc. 16 (1965), 987-990.

The Ohio State University 